\title{
Introduciendo SigProU, una interfaz gráfica de usuario para el análisis y procesamiento de señales EEG
}

\author{
Alina Santillán Guzmán \\ alina.santillan@upaep.mx \\ Azael Campeche Ramírez \\ azael.campeche@upaep.edu.mx \\ Universidad Popular Autónoma del Estado de Puebla \\ Puebla - México
}

\section{RESUMEN}

Las señales electroencefalográficas (EEG) suelen ser procesadas digitalmente usando diversos softwares como Python y MATLAB. En este artículo se presenta una interfaz gráfica de usuario para llevar a cabo el filtrado pudiendo elegir filtros pasa-bajas, pasaaltas, pasa-banda y rechaza-banda en diferentes aproximaciones (Butterworth, Chebyshev I, Chebyshev II o inverso, Elíptico). Asimismo, es posible realizar el análisis en tiempo (calculando la varianza, curtosis, correlación) y frecuencia (obteniendo la densidad espectral de potencia y la coherencia), así como el despliegue de mapas topográficos de señales EEG. La interfaz gráfica, llamada SigProU, ha sido desarrollada en MATLAB y se ha realizado la versión ejecutable para que pueda ser usada en cualquier computadora, aún sin tener el software instalado. SigProU es una interfaz amigable e intuitiva y fue comparada con EEGLAB, una interfaz comúnmente usada para el procesamiento de señales EEG. Es conveniente mencionar que no se pretende competir con dicha interfaz, sino más bien mostrar una herramienta más para el procesamiento de las señales EEG.

Palabras clave: interfaz gráfica de usuario; EEG; procesamiento. 


\title{
Introducing SigProU, a graphical user interface to analyze and process EEG signals
}

\begin{abstract}
Electroencephalographic (EEG) signals are used to be digitally processed using software such as Python and MATLAB. In this paper a graphical user interface is presented, which is used to filter the signals by means of a low-pass, high-pass, band-pass and stop-band filters, in different approximations (Butterworth, Chebyshev I, Chebyshev II or inverse Chebyshev, Elliptic). Moreover, it is possible to analyze the signals in time (computing the variance, kurtosis, correlation) and frequency (computing the power spectral density and coherence), and also the topographic maps of the EEG signals are displayed. The graphical user interface, called SigProU, has been developed in MATLAB and a exe version has been created in order to use the interface in any computer, even if MATLAB is not installed. SigProU is an intuitive and friendly interface and has been compared to EEGLAB, which is commonly used to process EEG signals. It is important to mention that it is not intended to compete against EEGLAB, but to show that SigProU can be one more tool to process the EEG signals.
\end{abstract}

Keywords: graphical user interface; EEG; processing

Artículo recibido: 02 noviembre. 2021 Aceptado para publicación: 28 noviembre 2021 Correspondencia: alina.santillan@upaep.mx Conflictos de Interés: Ninguna que declarar 


\section{INTRODUCCIÓN}

Las señales electroencefalográficas (EEG) son usadas para diagnosticar o analizar la evolución de diferentes desórdenes o enfermedades neurológicas, como el Parkinson o la epilepsia. Asimismo, también son usadas para determinar los diferentes estados que los sujetos de prueba tienen mientras hacen alguna actividad o son estimulados. Las señales EEG, también llamadas ritmos cerebrales, tienen un rango de frecuencia de 0.1 a $100 \mathrm{~Hz}$, siendo el ritmo delta el más lento (0.1-4 Hz) y gamma el más rápido (30-100Hz) (Sanei y Chambers, 2007). Dichas señales son usualmente contaminadas con artefactos (distorsiones) fisiológicos (movimientos oculares, musculares, ritmo cardiaco) o técnicos (línea de alimentación) (Sanei y Chambers, 2007; Ille, 2001), los cuales deberán eliminarse en la medida de lo posible, ya que suelen ocultar la información cerebral a ser analizada posteriormente. Para ello, diferentes tipos de filtros suelen usarse. Por ejemplo, existe el filtro pasa-bajas, pasa-banda o rechaza-banda para eliminar artefactos musculares, dejar pasar solo un rango de frecuencias o para eliminar el artefacto de la línea de alimentación, respectivamente (Malvino, 2000). Existen otras técnicas más complejas para poder eliminar artefactos, tales como el análisis de componentes independientes (ICA, por sus siglas en inglés) (Hyvärinen y Oja, 2000; Hyvärinen, 1999; Vigário, Särelä, Jousmäki, Hämäläinen y Oja, 2000) o descomposición empírica de modos (EMD) (Huang et al. 1998). Además de llevar a cabo un filtrado en las señales, también es necesario analizarlas en tiempo y en frecuencia. Para ello, se pueden obtener medidas estadísticas como la varianza, la curtosis, e incluso calcular la correlación que existe entre dos diferentes señales (canales o electrodos). De esta manera se puede llevar a cabo la caracterización de señales en el dominio del tiempo. Mientras tanto, para el análisis en frecuencia, es posible obtener la densidad espectral de potencia, con la cual se pueden visualizar las frecuencias características de las señales y determinar cuáles corresponden a señales deseadas y cuáles se tendrían que eliminar. Además, se puede obtener la potencia por bandas, dependiendo cuál se pretenda analizar. El cálculo de la potencia de banda es usado para obtener los mapas topográficos y con ello visualizar cuáles zonas cerebrales están más activadas.

Existen diferentes herramientas gráficas que ayudan al procesamiento y visualización de las señales EEG. Una de ellas es TMSEEG, la cual es una interfaz gráfica de usuario para el procesamiento de señales electrofisiológicas durante estimulación magnética 
transcraneal (TMS). Dicha interfaz pretende promover la gran utilidad de este tipo de herramientas para la investigación del cerebro (Atluri et al., 2016). Otra interfaz gráfica de usuario, implementada en MATLAB, fue creada para detectar los parpadeos. En esta interfaz, se seleccionan los canales, se filtran y se promedian las señales para lograr la detección (Selvi, Ferıkoğlu, Güzel y Karagöz, 2017). También se pueden encontrar interfaces específicas para filtrado, como por ejemplo fasticag (FastICA online, 2018) la cual sirve para hacer una descomposición de las señales EEG y eliminar diferentes tipos de artefactos. Otra interfaz de uso específico es HERMES (Niso et al., 2013), la cual mide la conectividad de las señales a través del cálculo de correlación, coherencia, sincronización en fase, causalidad de Granger, entre otros.

Una de las herramientas más comunes y utilizadas para el procesamiento de señales EEG es EEGLAB (Delorme y Makeig, 2004), con la cual se pueden filtrar las señales con filtros de tipo FIR (Finite Impulse Response), con ICA, seleccionar canales o componentes a analizar y obtener sus características espectrales a través de mapas topográficos.

En el presente trabajo se introduce una nueva herramienta llamada SigProU, con la cual se pueden filtrar señales EEG, analizar en tiempo (cálculo de varianza, curtosis, correlación) y frecuencia (Coherencia, densidad espectral de potencia, espectrograma), así como el despliegue de mapas topográficos en las diferentes bandas (theta:4-7 Hz, alpha: 8-12 Hz, Beta: 12-30 Hz). Cabe mencionar que esta nueva interfaz gráfica de usuario está en español y es de uso académico y no pretende competir con EEGLAB, sino más bien mostrar que puede ser una herramienta más para poder llevar a cabo el análisis y procesamiento de señales EEG de una forma intuitiva y amigable.

En la sección 2 se presentan de forma teórica lo que se ha implementado en esta interfaz. La sección 3 muestra los resultados tras hacer la implementación en MATLAB y se presentan ejemplos de su uso y una comparación de funcionamiento con respecto a EEGLAB. La sección 4 presenta las conclusiones y el trabajo a futuro a desarrollar.

\section{INTERFAZ GRÁFICA DE USUARIO SIGPROU}

A continuación, se presenta la descripción teórica de los diferentes tipos de filtros digitales implementados en la interfaz gráfica de usuario, así como los cálculos estadísticos y en frecuencia y los mapas topográficos. Cabe mencionar que, en cualquiera 
de las secciones de la interfaz, es posible guardar las señales originales, filtradas, así como los cálculos realizados.

\subsection{Filtrado}

Un filtro digital es un conjunto de operaciones que se le aplican a una señal de entrada para generar una señal de salida, atenuando ciertas frecuencias y dejando pasar las que resulten de interés. En la sección llamada "Filtrado" dentro de SigProU se encuentra una serie de filtros (pasa-bajas, pasa-altas, pasa-banda y rechaza-banda) en diferentes aproximaciones (Butterworth, Chebyshev I, Chebyshev II o inverso y Elíptico), con los cuales las señales EEG seleccionadas (puede ser solo una o pueden ser varias) son filtradas y de esta manera eliminar diferentes tipos de artefactos o solamente dejar pasar el rango de frecuencias deseado para su posterior análisis.

Los filtros pasa-bajas, como su nombre lo indica, dejarán pasar las bajas frecuencias, desde cero hasta una frecuencia de corte determinada, conocida como banda-pasante, y atenuarán el resto (banda-eliminada). Los filtros pasa-altas dejarán pasar las altas frecuencias desde una frecuencia de corte en adelante. Los filtros pasa-bandas tendrán dos frecuencias de corte, la inferior y superior, teniendo así un ancho de banda, la cual será lo que dejarán pasar, atenuando las demás frecuencias que no estén incluidas dentro de esta banda. Mientras que los filtros rechaza-banda dejarán pasar solamente un rango de frecuencias que estén de 0 a la frecuencia de corte inferior y de la frecuencia de corte superior en adelante; rechazando las frecuencias que estén entre la inferior y superior.

Idealmente, la región de transición entre la banda pasante y la banda eliminada es vertical. Sin embargo, en la práctica esto no sucede y por lo tanto es necesario hacer aproximaciones para llevar a cabo el filtrado lo más cercano a la forma ideal.

\subsubsection{Aproximación Butterworth}

Los filtros Butterworth se caracterizan por tener una amplitud máximamente plana en la banda pasante y una caída monotónica en la banda eliminada. La pendiente decae a razón de $20 \mathrm{n} \mathrm{dB/década} \mathrm{o} 6 \mathrm{n} \mathrm{dB/octava,} \mathrm{siendo} \mathrm{n} \mathrm{el} \mathrm{orden} \mathrm{del} \mathrm{filtro} \mathrm{(Malvino,} \mathrm{2000).}$

\subsubsection{Aproximación Chebyshev I}

El filtro Chebyshev I, o simplemente Chebyshev, presenta rizos en la banda pasante, lo cual conlleva a amplitudes que fluctúan en las señales filtradas en las frecuencias de la banda-pasante. Sin embargo, la ventaja de este filtro es que su pendiente de caída es más 
rápida que los filtros Butterworth por 6(n-1) dB (Malvino, 2000). La amplitud de los rizos está dada en $\mathrm{dB}$ y depende del usuario seleccionar su valor.

\subsubsection{Aproximación Chebyshev II}

También llamado Chebyshev inverso, presenta rizos en la banda-eliminada, y una amplitud plana en la banda-pasante (Malvino, 2000). El valor del rizo en la bandaeliminada está dado en $\mathrm{dB}$ y es definido por el usuario. La ventaja de este filtro es que no habrá atenuaciones en la banda pasante, pero la desventaja es que habrá amplitudes oscilantes en la señal filtrada en las frecuencias de la banda-eliminada.

\subsubsection{Aproximación Elíptica}

Este tipo de aproximación presenta rizos tanto en la banda pasante como en la banda eliminada, por lo que habrá atenuaciones en la señal de salida en las frecuencias de la banda pasante y habrá amplitudes oscilantes en la señal filtrada en las frecuencias de la banda-eliminada (Malvino, 2000). La ventaja de esta aproximación es que se logra un diseño eficiente con un orden menor, comparado con las demás aproximaciones.

En todos los tipos de filtro implementados en SigProU y sus correspondientes aproximaciones, es posible que, dependiendo de los requerimientos del usuario respecto al filtrado, se elijan tanto el tipo de filtro, la aproximación que considere conveniente, así como los parámetros de entrada para el filtro elegido (valor de rizos, frecuencia(s) de corte, orden y la señal o señales a ser filtradas).

\subsection{Análisis en Tiempo}

En la sección "Análisis" es posible hacer éste en el tiempo y en la frecuencia. En el análisis en el dominio del tiempo se calcula la varianza, la cual es una medida de dispersión que en señales EEG es usada para determinar si existe alguna distorsión o algún evento (por ejemplo, epiléptico), ya que el valor de la varianza en estos casos suele ser alta, en comparación con señales no distorsionadas o consideradas como normales. Asimismo, se calcula la curtosis, la cual también es una medida estadística que mide la distribución de la señal. Si la distribución es Gaussiana, la curtosis es cero. Si la distribución es superGaussiana, la curtosis es positiva y si la distribución es sub-Gaussiana, la curtosis es negativa (Heumann, Schomaker y Shalabh, 2016). La curtosis también es empleada para determinar diferentes tipos de distorsiones o la presencia de algún evento en las señales EEG. 
Otro cálculo que se realiza en el dominio del tiempo es el de la correlación, la cual es la relación de similitud que existe entre dos canales o señales. Si su valor es muy cercano a \pm 1 entonces existe una muy alta similitud entre las dos señales analizadas (Van Drongelen, 2007).

Para el caso de la varianza y la curtosis, el usuario puede seleccionar el número de segundos por segmento que quiera o necesite para calcular ambas medidas. Además, se obtiene el promedio de la varianza y curtosis por todos los segmentos calculados.

Para el caso de la correlación, el usuario selecciona los canales que quiere analizar, selecciona también el número de segundos por segmento para obtener la correlación, el número de "lags" y el tipo de normalización a ser calculada. Se muestra el promedio de la correlación calculada por todos los segmentos.

\subsection{Análisis en Frecuencia}

Además de poder analizar las señales EEG en el dominio del tiempo, se pueden analizar en el dominio de la frecuencia.

En la interfaz SigProU es posible obtener el Espectro de Potencia o la Densidad Espectral de Potencia de la señal o señales elegida(s) por el usuario. El espectro de potencia es usado para obtener las frecuencias que caracterizan a la señal analizada. Para ello, es necesario obtener la Transformada de Fourier de la señal en el tiempo y multiplicarla por su conjugado para obtener la potencia. De esta manera, es posible visualizar el espectro y determinar qué frecuencias tienen mayor o menor potencia, y por ende proporcionan información sobre señales o posibles distorsiones en las mismas.

Asimismo, es posible calcular la Coherencia, que es la medida de qué tan similares son dos señales en determinadas frecuencias. Mientras más cercano a 1 es el valor de la coherencia, mayor similitud habrá de las señales en dicha frecuencia (Van Drongelen, 2007).

El Espectrograma también es obtenido y graficado en la interfaz. El espectrograma es la representación de la potencia (dependiendo el color) en tiempo y frecuencia de la señal a ser analizada. Se calcula usando la transformada de Fourier por ventanas. La longitud de cada ventana es elegida por el usuario.

\subsection{Mapas Topográficos}

En la sección de "Mapas" es posible observar la representación de la cabeza iluminada en diferentes colores indicando qué tanta actividad está sucediendo en las distintas áreas 
cerebrales. El color rojo indica mucha actividad, mientras que color azul indica que la actividad es casi nula.

Se usa uno de los modelos válidos para la representación de la superficie de la cabeza, el modelo plano (Mecarelli, 2019). El modelo permite la localización de los electrodos con coordenadas X Y. Con las coordenadas y la información del EEG, se aplica una interpolación de spline (Christoph, Koening, Brandeis, Gianotti y Wackermann, 2009). Esto permite tener información para poder representar la menor y mayor actividad cerebral.

Los mapas topográficos son obtenidos usando la potencia existente en cierto intervalo y se grafica por cada ritmo cerebral. Así, es posible observar el mapa de la onda delta, theta, alpha, beta y gamma. El usuario puede elegir una o más bandas a observar.

\section{RESULTADOS Y DISCUSIÓN}

En la Fig. 1 se muestra el Menú Principal de la interfaz SigProU. Ahí se muestran las tres secciones descritas anteriormente. Cuando el usuario da click en cada una de ellas, se muestra una pequeña descripción de lo que se podrá hacer en cada caso.

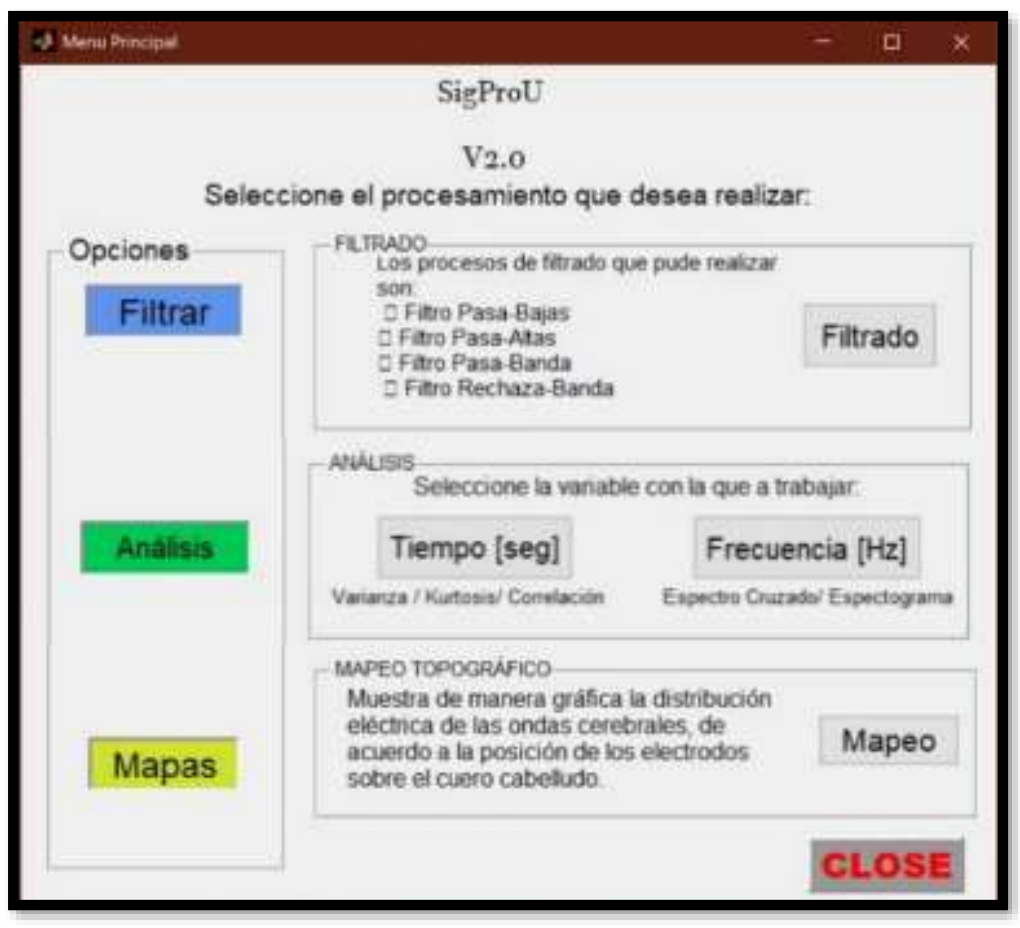

Figura 1. Menú Principal de la interfaz gráfica SigProU

Cuando el usuario elige cualquiera de las opciones, tiene que elegir la serie de datos que utilizará, así como el sistema con el que fueron adquiridas las señales para poder elegir 
los canales. En la interfaz están los sistemas del EMOTIV Epoc+, BESA, configuraciones de 25, 32, 64, 75 canales y personalizado. En este último, el usuario puede usar un archivo de formato específico, para trabajar con una configuración de canales que no se encuentre dentro de la interfaz. En el formato, se requiere colocar las coordenadas esféricas de los electrodos que se usaron. El usuario seleccionará aquél que corresponda a las señales a analizar y asimismo el o los canales que procesará o analizará posteriormente. En la Fig. 2 se muestra un ejemplo de cómo se ve esta etapa, donde se observa el sistema EMOTIV del Epoc+.

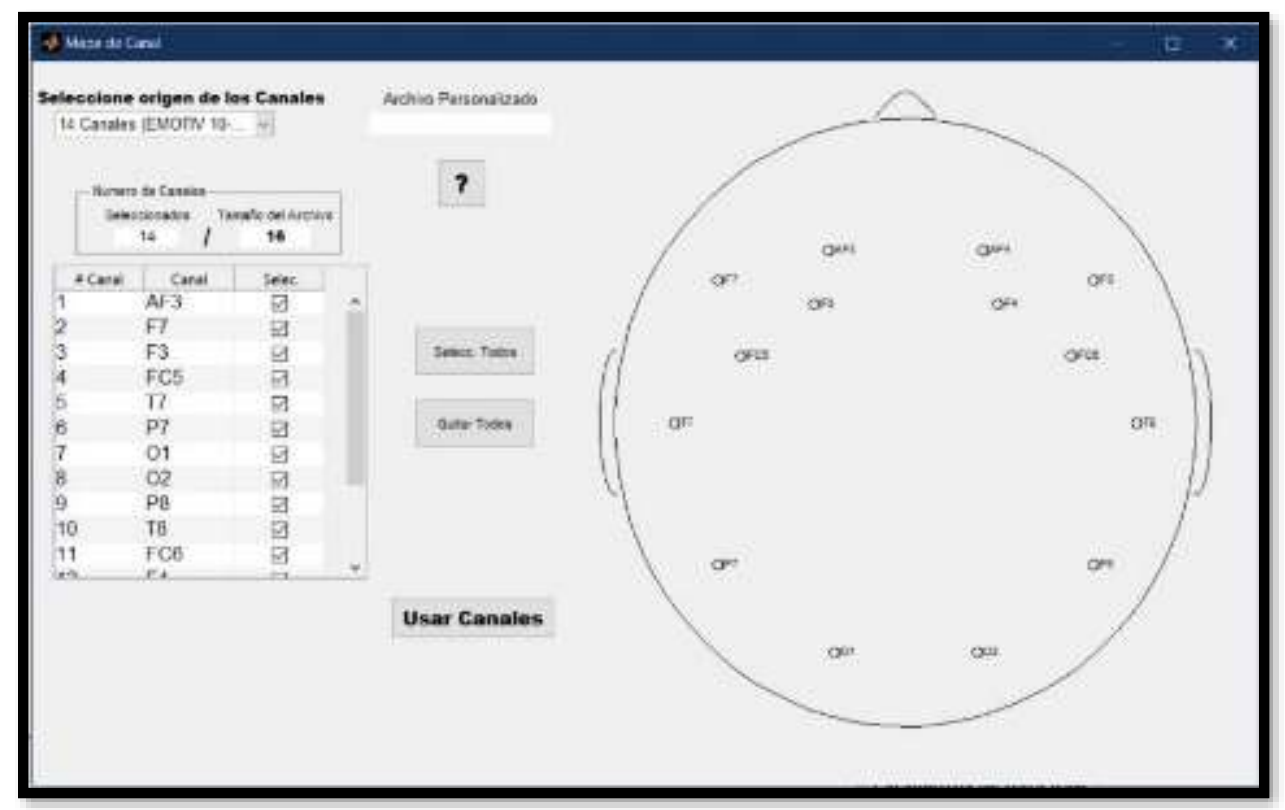

Figura 2. Ejemplo del sistema de posicionamiento del EMOTIV Epoc+ y los canales a seleccionar para ser analizados y procesados en la interfaz gráfica.

Una vez elegidos los canales y las señales a ser analizadas, y previo al filtrado, se pueden graficar las señales originales en el tiempo, tal como se observa en la Fig. 3, donde el eje $x$ corresponde al tiempo en segundos y el eje $y$ es cada canal con su correspondiente etiqueta. Con los símbolos " $<<" \mathrm{y}$ " $>>$ " se pueden ir pasando $20 \mathrm{~s}$ hacia atrás o hacia adelante, respectivamente. Con los símbolos "<" y "> " se ve un segundo menos o un segundo más, respectivamente en cada ventana. También se pueden escribir los parámetros de los datos crudos, como la frecuencia de muestreo, la longitud de la ventana que se quiere visualizar, el tiempo de inicio donde comenzará la muestra, un acercamiento y un alejamiento para mejorar la visualización de la muestra. Como ejemplo se despliegan $20 \mathrm{~s}$ de señales EEG de un sujeto sano, con una frecuencia de muestreo de $128 \mathrm{~Hz}$. 
En la Fig. 4 se están graficando las mismas señales EEG usando EEGLAB a manera de comparación con las mostradas en SigProU. Como se puede observar, en ambas interfaces las señales lucen muy similares, lo cual indica que la interfaz propuesta es comparable con la ya existente.

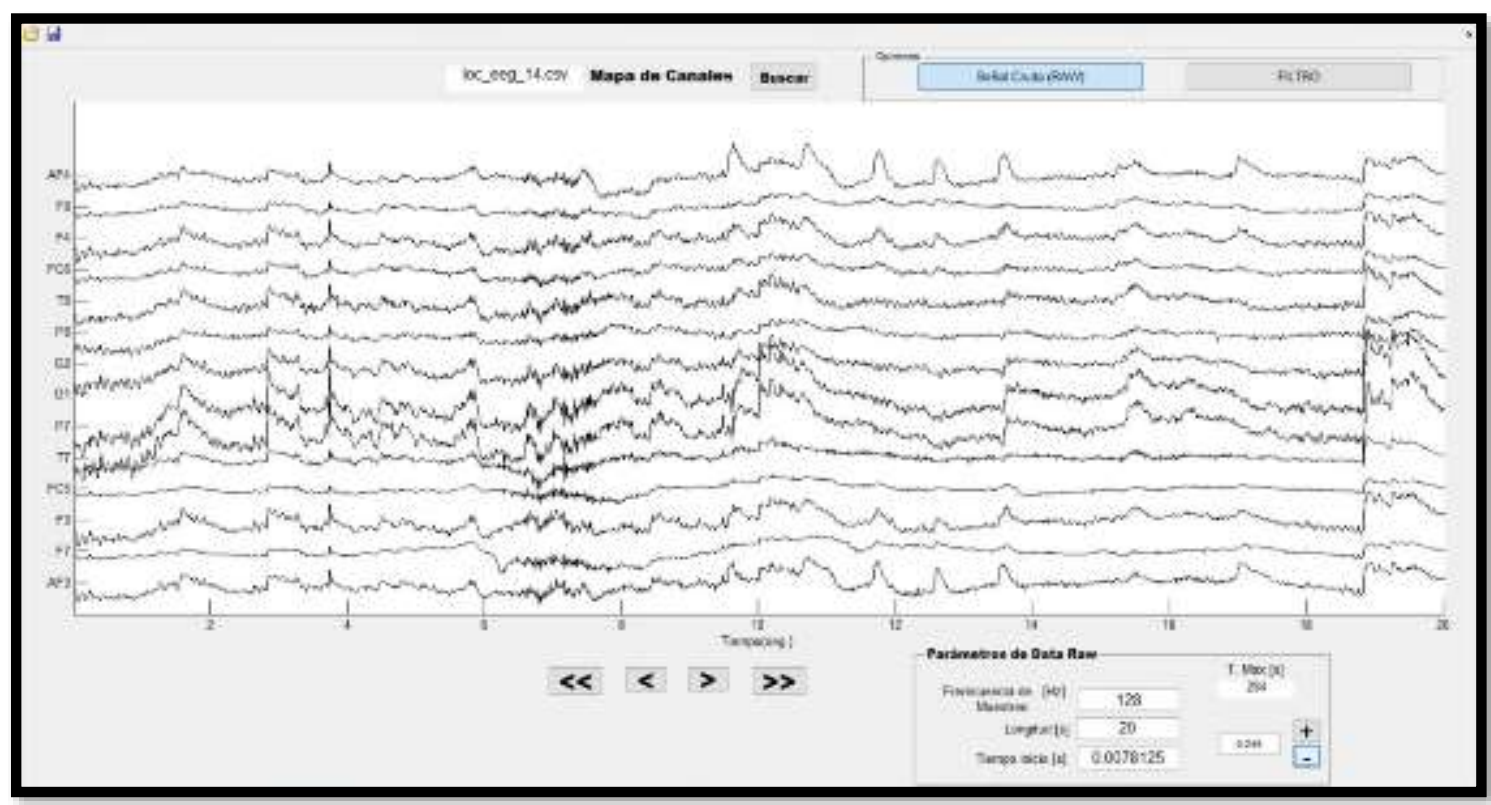

Figura 3. Visualización de las señales originales previo al filtrado, usando SigProU.

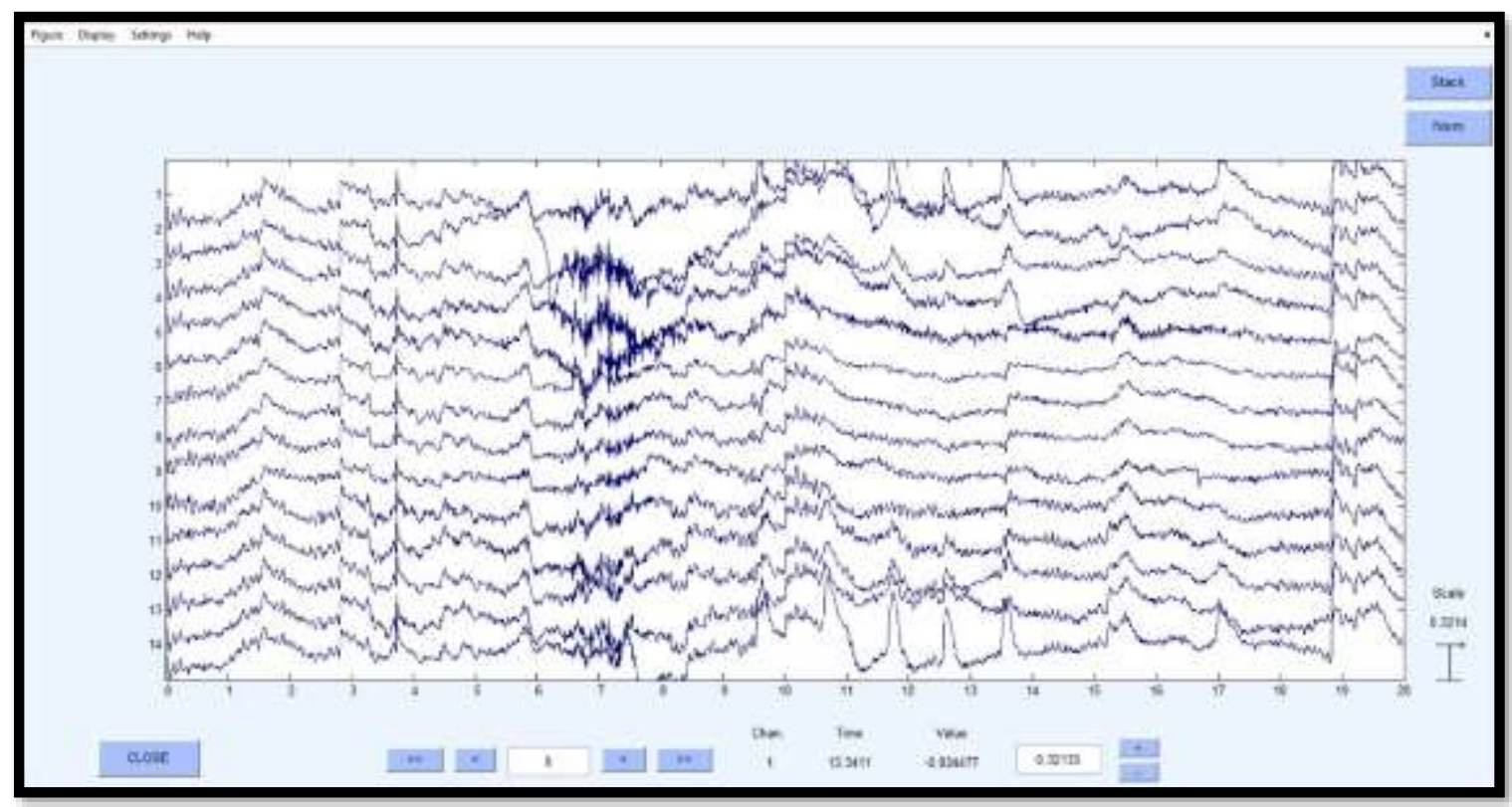

Figura 4. Visualización de las señales originales usando EEGLAB 
En la Fig. 5 se muestra un ejemplo de la interfaz de filtrado con un filtro pasa-bajas Butterworth de cuarto orden y cuya frecuencia de corte es $15 \mathrm{~Hz}$. En la parte izquierda se presentan todos los tipos y aproximaciones de filtros que pueden usarse. En la parte superior derecha se grafican las señales originales y filtradas y en la parte inferior derecha la densidad espectral de potencia de la señal original y filtrada del canal que seleccione el usuario. En el ejemplo puede observarse que el filtro está funcionando correctamente, al eliminar las frecuencias mayores a $15 \mathrm{~Hz}$ y dejando pasar las bajas frecuencias.

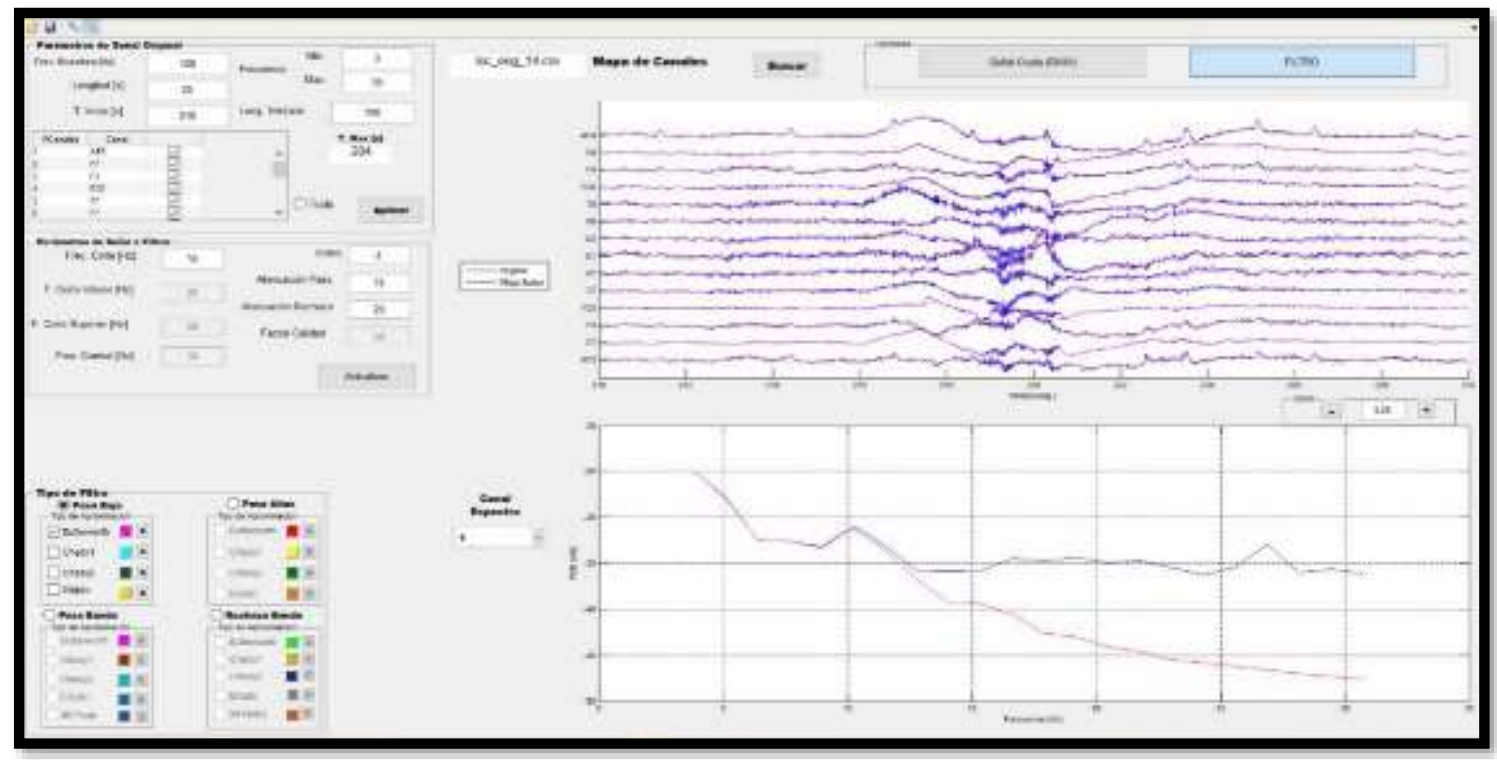

Figura 5. Ejemplo de señales filtradas usando un filtro pasa-bajas Butterworth de cuarto orden y frecuencia de corte de $15 \mathrm{~Hz}$, usando la interfaz SigProU.

En la Fig. 6 se muestra la interfaz de análisis en el dominio del tiempo. Como se puede observar en la parte superior izquierda está el panel donde se ingresan los parámetros de las señales, tales como frecuencia de muestreo, longitud e inicio de la señal a analizar, canales a elegir. En el siguiente panel (centro izquierdo) se puede escribir el número de segundos por segmento para calcular la varianza y la curtosis, así como el canal al que se quiere hacer los cálculos. Asimismo, se despliegan los resultados del promedio de la varianza y curtosis. En el siguiente panel se eligen los canales a correlacionar seleccionando el tipo de normalización para el cálculo de la correlación, el número de lags y los segundos por segmento. En la parte derecha se muestran las señales originales (parte superior), los resultados de la varianza y curtosis por cada segmento elegido (parte central) y posteriormente los canales a correlacionar y su correspondiente valor de correlación. 


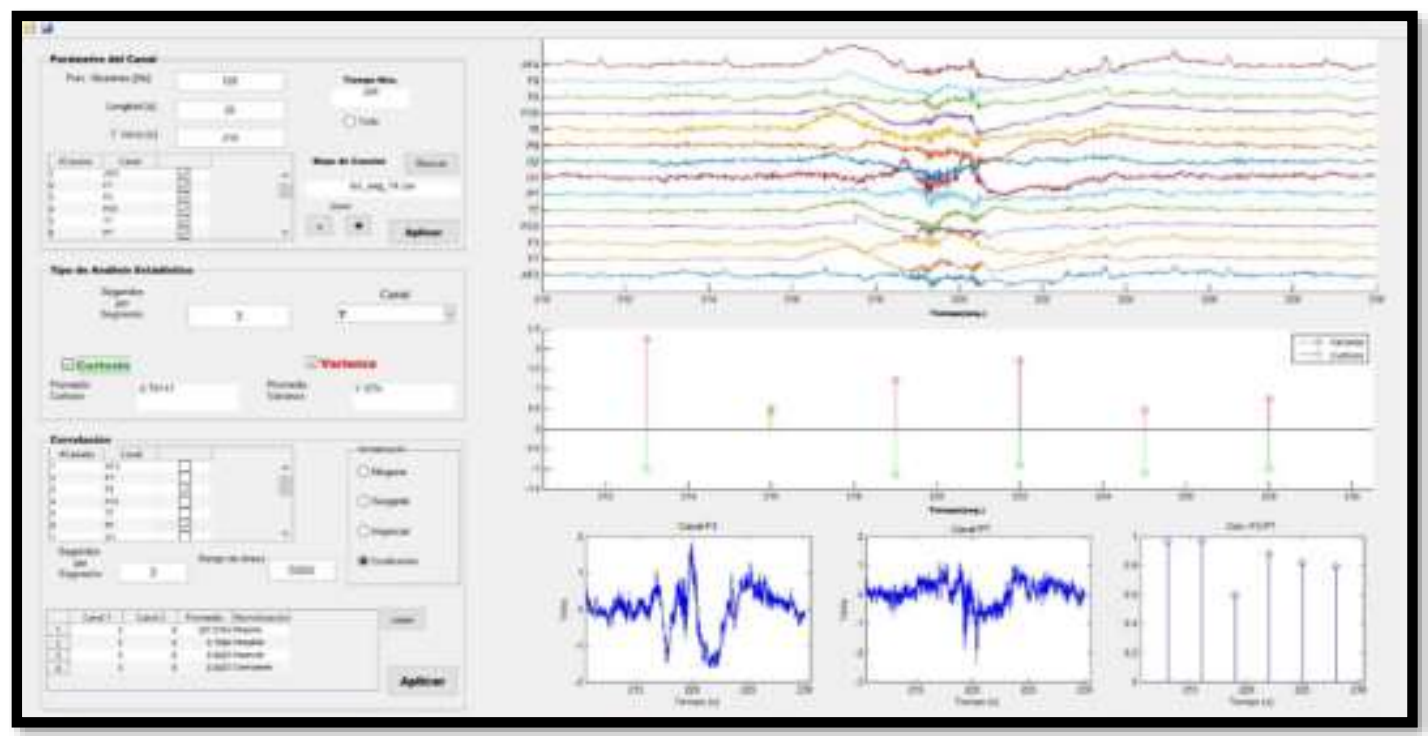

Figura 6. Ejemplo de la interfaz de análisis en el dominio del tiempo.

A manera de ejemplo de la interfaz de análisis en la frecuencia, se presenta el resultado de la coherencia entre dos canales seleccionados, tal como se observa en la Fig. 7. En dicha interfaz, además de ingresar la frecuencia de muestreo, longitud e inicio de la señal a analizar, también se escriben las frecuencias mínima y máxima a observar en el espectro de potencia. Asimismo, es posible escribir la longitud de la ventana para calcular el espectrograma y la coherencia. Se puede hacer un acercamiento a cada figura, elegir los canales para graficar su correspondiente espectro, entre otros.

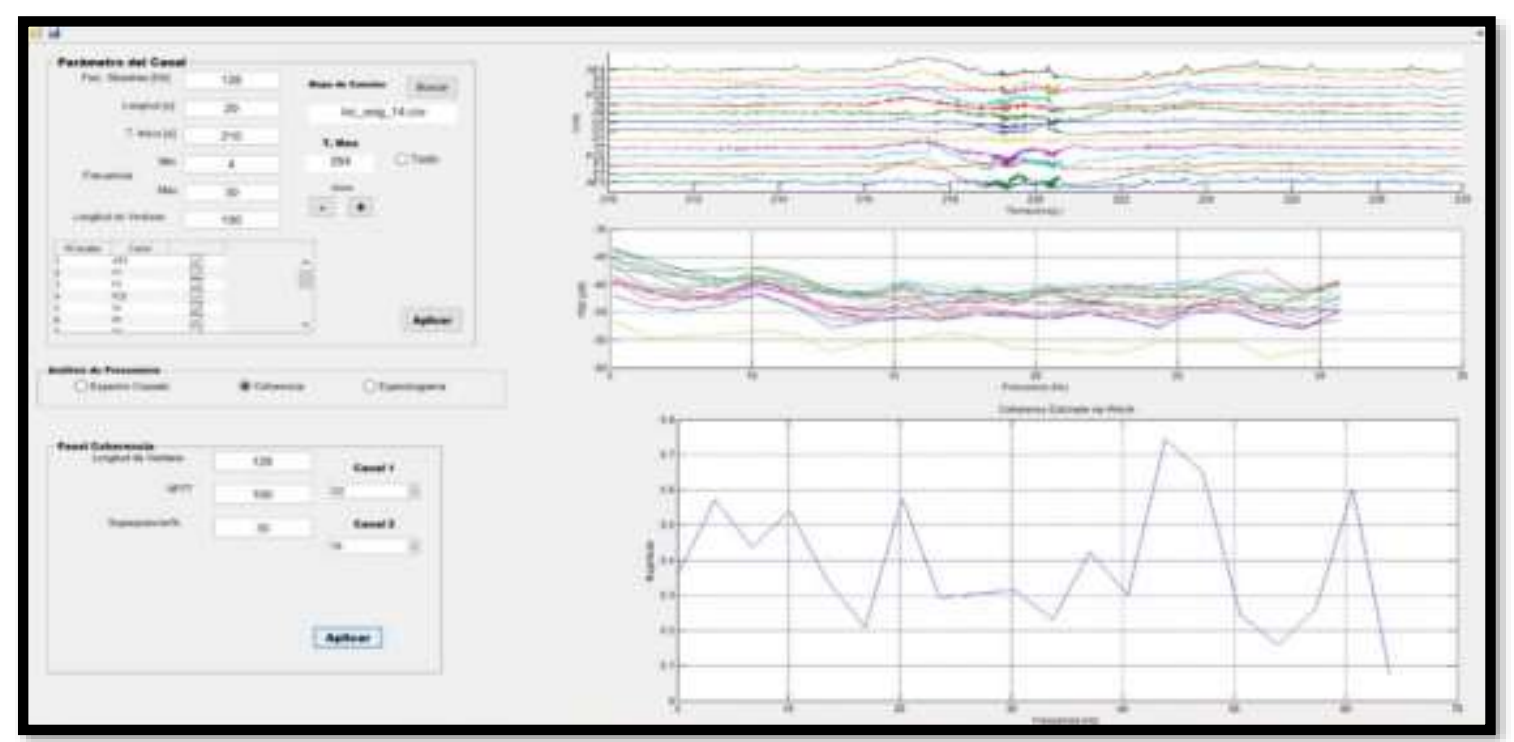

Figura 7. Interfaz de análisis en el dominio de la frecuencia. Ejemplo para la Coherencia. 
En la Fig. 8 se muestra la interfaz de Mapas, en el ejemplo se muestran los diferentes mapas topográficos de todas las bandas de los ritmos cerebrales. En la parte derecha se muestra el mapa topográfico correspondiente al punto indicado por la línea (aproximadamente 8.5 segundos después de iniciado el análisis). En la Fig. 9 se muestra el mapa topográfico obtenido con EEGLAB en el mismo punto que con SigProU. Como se puede observar, en ambas imágenes se tiene una mayor actividad en la parte occipital izquierda. Esto quiere decir que los resultados de SigProU son comparables con los de EEGLAB.

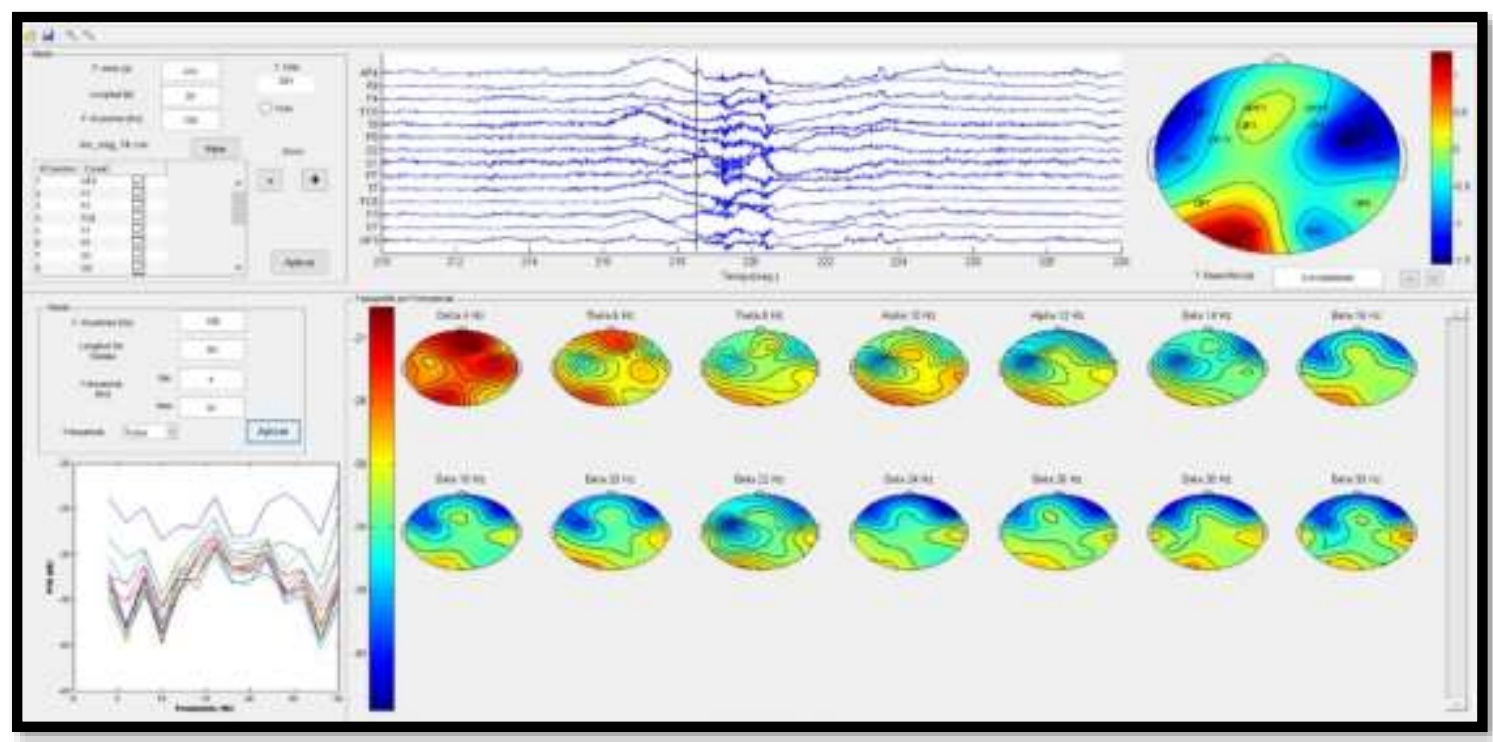

Figura 8. Interfaz Mapas mostrando un ejemplo con las señales EEG. Se pueden observar los mapas topográficos en las diferentes bandas de frecuencias.

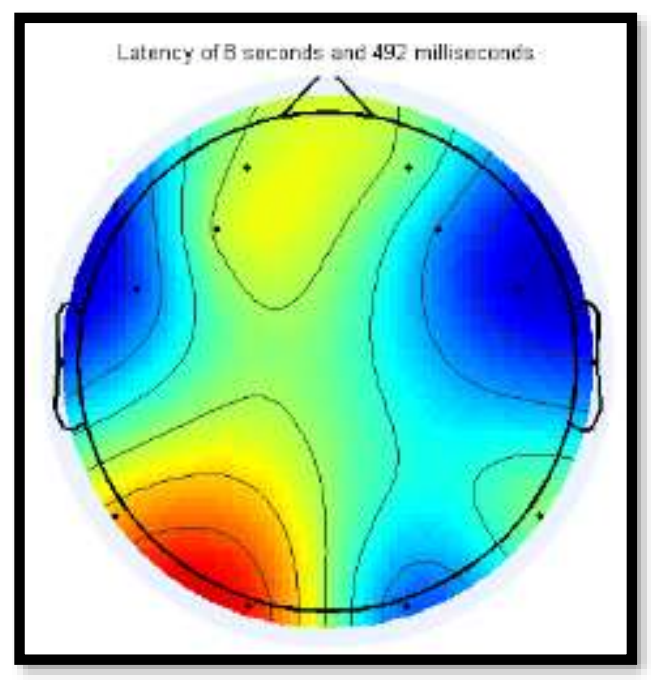

Figura 9. Mapa topográfico tomado 8.492 segundos después de iniciado el análisis, usando EEGLAB. Se observa mayor actividad en la parte occipital izquierda. 


\section{CONCLUSIÓN}

En el presente trabajo se describió la interfaz gráfica SigProU, la cual puede ser utilizada como herramienta alternativa académica para el procesamiento de señales EEG. Es una interfaz gráfica amigable e intuitiva implementada en MATLAB. A través de ejemplos se mostró el correcto funcionamiento de cada sección de la interfaz: Filtros, Análisis (en tiempo y frecuencia) y Mapas topográficos. Asimismo, se hizo una comparación con EEGLAB y se demostró que el funcionamiento de SigProU es comparable con éste, especialmente en los Mapas Topográficos, tomando en cuenta que en la interfaz propuesta se pueden observar más mapas según las bandas de los ritmos cerebrales, lo cual representa más información que se puede obtener de la interfaz.

Los parámetros de entrada pueden ser elegidos y escritos por el usuario y en caso de que éste cometa un error, mensajes de advertencia aparecen para que rectifique.

Se creó una versión ejecutable para que pueda ser usada en cualquier computadora sin necesidad de instalar el software y con ello cualquier usuario puede emplear esta interfaz. El idioma de SigProU es el español facilitando de esta manera su uso para estudiantes e investigadores de habla hispana, sin embargo, como trabajo futuro se pretende hacer la misma interfaz en inglés para que pueda ser usada por más personas. Además, se integrarán más secciones, especialmente para la detección semi-automática de artefactos y su eliminación.

\section{LISTA DE REFERENCIAS}

Atluri, S., Frehlich, M., Mei, Y., García L., Rogasch, NC., Wong, W., Daskalakis ZJ. y Farzan, F. (2016). TMSEEG: A MATLAB-Based Graphical User Interface for Processing Electrophysiological Signals during Transcranial Magnetic Stimulation. Front Neural Circuits, 7, 10-78. https://doi.org/10.3389/fncir.2016.00078

Christoph, M., Koening, T., Brandeis, D., Gianotti, L. y Wackermann, J. (2009), Electrical Neuroimaging, Cambridge.

Delorme, A. y Makeig, S. (2004). EEGLAB: an open source toolbox for analysis of single-trial EEG dynamics including independent component analysis. J Neurosci Methods, 134(1), 9-21. https://doi.org/10.1016/j.jneumeth.2003.10.009

FastICA. FastICA MATLAB package [Internet]. 2018, Nov. Available from: https://research.ics.aalto.fi/ica/fastica/code/dlcode.shtml 
Heumann, C., Schomaker, M. y Shalabh. (2016). Measures of Central Tendency and Dispersion. Introduction to Statistics and Data Analysis (pp. 50-52). Suiza,Springer Nature.

Huang, NE et al. (1998). The empirical mode decomposition and Hilbert spectrum for nonlinear and non-stationary time series analysis. Proceedings of the Royal Society of London. Series A: Mathematical, Physical and Engineering Sciences, 454, 903-995. https://doi.org/10.1098/rspa.1998.0193

Hyvärinen, A. y Oja, E. (2000). Independent component analysis: Algorithms and applications. Neural Networks 13(4-5), 411-430. doi: https://doi.org/10.1016/S0893-6080(00)00026-5.

Hyvärinen, A. (1999). Fast and Robust Fixed-Point Algorithms for Independent Component Analysis. IEEE on Transactions on Neural Networks, 10(3), 626-634. https://doi.org/10.1109/72.761722

Ille, N. (2001). Artifact correction in continuous recordings of the electro-and magnetoencephalogram by spatial filtering ( $\mathrm{PhD}$ thesis). Universität Mannheim.

Malvino, A. P. (2000). Principios de Electrónica. España: Mc Graw Hill.

Mecarelli, O. (2019) . Clinical Electroencephalography, Springer.

Niso, G. et al. (2013) HERMES: towards an integrated toolbox to characterize functional and effective brain connectivity. Neuroinformatics, 11(4), 405-434, https://doi.org/10.1007/s12021-013-9186-1

Sanei, S., y Chambers, J. (2007). EEG Signal Processing. England: John Wiley \& Sons Ltd. https://doi.org/10.1002/9780470511923.

Selvı A. O., Ferıkoğlu, A., Güzel, D. y Karagöz, E. (2017). Design and implementation of EEG signal based brain computer interface for eye blink detection. 2017 International Conference on Computer Science and Engineering (UBMK), 544548. https://doi.org/10.1109/UBMK.2017.8093458

Van Drongelen, W. (2007). LTI Systems, Convolution, Correlation, and Coherence. Signal Processing for Neuroscientists (pp. 136-142). Elsevier

Vigário, R., Särelä, J., Jousmäki, V., Hämäläinen, M. y Oja, E. (2000). Independent component approach to the analysis of EEG and MEG recordings. IEEE Transactions on Biomedical Engineering, 47(5), 589-593. https://doi.org/10.1109/10.841330 